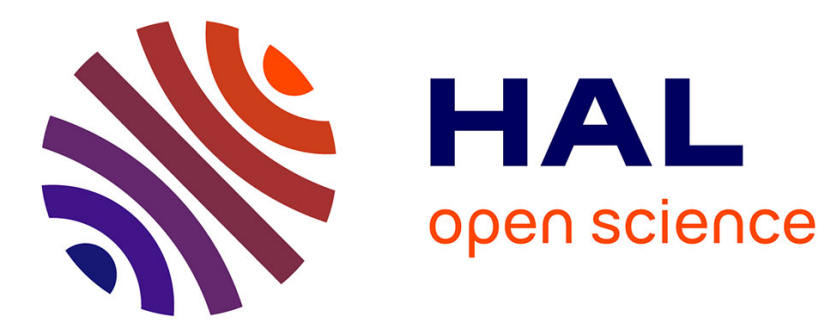

\title{
Prevention of treatable infectious diseases: a game-theoretic approach
}

Sofía Jijón, Virginie Supervie, Romulus Breban

\section{To cite this version:}

Sofía Jijón, Virginie Supervie, Romulus Breban. Prevention of treatable infectious diseases: a gametheoretic approach. Vaccine, 2017, 35, pp.5339-5345. 10.1016/j.vaccine.2017.08.040 . pasteur01634523

\section{HAL Id: pasteur-01634523}

\section{https://hal-pasteur.archives-ouvertes.fr/pasteur-01634523}

Submitted on 14 Nov 2017

HAL is a multi-disciplinary open access archive for the deposit and dissemination of scientific research documents, whether they are published or not. The documents may come from teaching and research institutions in France or abroad, or from public or private research centers.
L'archive ouverte pluridisciplinaire HAL, est destinée au dépôt et à la diffusion de documents scientifiques de niveau recherche, publiés ou non, émanant des établissements d'enseignement et de recherche français ou étrangers, des laboratoires publics ou privés.

\section{다)(1) $(5$}

Distributed under a Creative Commons Attribution - NonCommerciall 4.0 International 


\title{
Prevention of treatable infectious diseases: a game-theoretic approach
}

\author{
Sofía Jijón ${ }^{\mathrm{a}, *}$, Virginie Supervie ${ }^{\mathrm{a}}$, Romulus Breban ${ }^{\mathrm{b}}$ \\ ${ }^{a}$ Sorbonne Universités, UPMC Univ Paris 06, INSERM, Institut Pierre Louis \\ d'Épidémiologie et de Santé Publique (IPLESP UMR_S 1136), Paris, France. \\ ${ }^{b}$ Institut Pasteur, Unité d'Epidémiologie des Maladies Emergentes, Paris, France.
}

\begin{abstract}
We model outcomes of voluntary prevention using an imperfect vaccine, which confers protection only to a fraction of vaccinees for a limited duration. Our mathematical model combines a single-player game for the individual-level decision to get vaccinated, and a compartmental model for the epidemic dynamics. Mathematical analysis yields a characterization for the effective vaccination coverage, as a function of the relative cost of prevention versus treatment; note that cost may involve monetary as well as non-monetary aspects. Three behaviors are possible. First, the relative cost may be too high, so individuals do not get vaccinated. Second, the relative cost may be moderate, such that some individuals get vaccinated and voluntary vaccination alleviates the epidemic. In this case, the vaccination coverage grows steadily with decreasing relative cost of vaccination versus treatment. Unlike previous studies, we find a third case where relative cost is sufficiently low so epidemics may be averted through the use of prevention, even for an imperfect vaccine. However, we also found that disease elimination is only temporary - as no equilibrium exists for the individual strategy in this third case - and, with increasing perceived cost of vaccination versus treatment, the situation may be reversed toward the epidemic edge, where the effective reproductive number is 1 . Thus, maintaining relative cost sufficiently low will be the main challenge to maintain disease elimination. Furthermore, our model offers insight on vaccine parameters, which are otherwise difficult to estimate. We apply our findings to the epidemiology of measles.
\end{abstract}

Keywords: prevention versus treatment, childhood infectious diseases, imperfect vaccine, SEIR model, game theory.

\footnotetext{
* Corresponding author

Email addresses: sofia.jijon@iplesp.upmc.fr (Sofía Jijón), virginie.supervie@inserm.fr (Virginie Supervie), romulus.breban@pasteur.fr (Romulus Breban)
} 


\section{Introduction}

The 20th century has witnessed tremendous achievements in infectious disease prevention, especially with the development of effective preventive vaccines [1], often far less costly than treatment [2]. Still, the preference between

5 prevention and treatment remains a dilemma. Some studies found no preference [3-5], others a preference for prevention $[6,7]$, or a preference for treatment $[8,9]$, or that preference for prevention versus treatment depends on the circumstances $[10,11]$.

The prevention of treatable infectious diseases still poses challenges for public health authorities [12]. Faced with infection risk, individuals may decide to use prevention, or else get treated if they acquired infection. Whereas treatment is generally well accepted by infected individuals, prevention may have a wide range of acceptability profiles for the susceptible. Individual-level perceptions of risk, as well as weighing pros and cons of prevention versus treatment, may 15 differ from the recommendations of the public health authority [13], for a variety of reasons $[14,15]$.

The decision to use voluntary vaccination and its impact on disease transmission has been theoretically studied using mathematical models with two components: one describing the population-level epidemiology and another describing the strategy by which an individual makes his choice of whether or not to get vaccinated [16-39]. Both compartmental models [16-18, 28, 33-39] and social networks [19-22] have been used as the population-level model component. For the individual-level component, imitation dynamics [22, 23, 39], "wait and see" strategies [38], social distancing strategies [24, 25], maximization of the utility of 25 prevention [16, 26-31] and inductive reasoning [33-36] have been studied. The role of altruism for the individual-level strategy has also been considered [32]. Several modeling studies discuss the impact of public misperceptions about vaccination programs on vaccination uptake $[16,22,23,27,28]$.

The main research direction of the modeling work has been individual and 30 group behavior in the dilemma of whether or not to get vaccinated [16-20, 22$32,37-39]$. Another direction has been vaccination subsidies and incentives [21, 33-36]. A review of recent literature can be found in Ref. [40].

The purpose of the current work is to assess the performance of a voluntary prevention program, utilizing an imperfect vaccine, which confers protection 35 only to a fraction of vaccinees for a limited duration. We show that voluntary vaccination with an imperfect vaccine may temporarily eliminate epidemics. We apply our findings to the measles epidemiology.

\section{Model}

We propose a mathematical model describing the interplay between volun-

40 tary vaccination and treatment during the course of an epidemic. In particular, our model addresses the setup where vaccination is available as a prevention method against childhood infectious diseases. However, we assume that the vaccine is imperfect $[41,42]$. We consider two aspects of vaccine failure and 
introduce appropriate parameters. First, the vaccine may not take for all vaccinees; the fraction of vaccinees for which the vaccine yields an immune response is called vaccine efficacy. This has been largely used to model voluntary vaccination $[27-29,32,38,39]$. In this case, the key epidemiological concept is the effective vaccination coverage [43, 44], the fraction of the population that acquires immunity due to vaccination. Second, even if the vaccinee acquires an

50 immune response, this may not result in lifelong immunity. That is, the vaccinee acquires a limited duration of immunity, a feature much less studied in the modeling of voluntary vaccination [20].

We describe epidemic dynamics using an SEIR-type system of ordinary differential equations. Recovery may be reached naturally or through treatment, 55 which may be either symptomatic or therapeutic. Furthermore, we involve an individual-level model of decision-making about whether or not to get vaccinated. We assume that individuals make their decisions by judging pros and cons for vaccination versus treatment, and have a sense of the imminence of getting infected and then treated. According to game theory, such a decision${ }_{60}$ making process may be modeled as a non-cooperative game, where individuals act in their own interest to maximize the utility of vaccination versus treatment. However, an individual's decision is indirectly influenced by those of others: the sum of all individuals' decisions determines the proportion of the population that gets vaccinated, which, in turn, affects the epidemic progression and the 65 probability of acquiring infection. The game model is intertwined with the model of epidemic dynamics. Model analyses assume that the resolution of the dilemma of vaccination versus treatment yields stable disease epidemiology.

\subsection{The compartmental model}

We make further assumptions for our deterministic SEIR-type model. The vaccination program is constantly in place, regardless of whether or not there is an epidemic. Treatment is available in unlimited supply, and no decision-making is involved about when to start treatment. Complete recovery is possible, with the benefit of lifelong immunity. These assumptions lead to the following ordinary differential equations of $S E I R$ type:

$$
\begin{aligned}
& \frac{d V}{d t}=\epsilon p \pi-(\rho+\mu) V, \\
& \frac{d S}{d t}=(1-\epsilon p) \pi+\rho V-\frac{\beta I}{N} S-\mu S, \\
& \frac{d E}{d t}=\frac{\beta I}{N} S-(\nu+\mu) E, \\
& \frac{d I}{d t}=\nu E-(\sigma+\gamma+\mu) I, \\
& \frac{d R}{d t}=(1-\xi) \sigma I+\gamma I-\mu R, \\
& \frac{d T}{d t}=\xi \sigma I-\mu T .
\end{aligned}
$$


Newborns can remain susceptible $(S)$ or acquire vaccine-induced immunity $(V)$, in which case they may become susceptible thereafter, as vaccine-induced immunity wanes. Recently infected individuals $(E)$ pass through a latent stage of infection. Then, they become infectious $(I)$ and can recover either naturally $(R)$ or through treatment $(T)$. The total population size is given by $N=$ 80 $V+S+E+I+R+T$.

The probability of getting vaccinated is denoted by $p$ and the vaccine parameters are $\epsilon$, the vaccine efficacy, and $\rho$, the rate of waning of vaccine-induced immunity. The parameter $\pi$ stands for the inflow of newborns, $\mu$ is the diseaseunrelated death rate, $\beta$ stands for the disease transmissibility, $\nu$ for the pro-

85 gression through the latency stage, $\sigma$ is the rate at which individuals start treatment, $\xi$ represents the treatment efficacy and $\gamma$ is the natural recovery rate. All variables and parameters are positively defined.

The model has two equilibria: a disease-free state (DFS) where

$$
V_{\mathrm{DFS}}=\frac{\epsilon p \pi}{\rho+\mu}, \quad S_{\mathrm{DFS}}=\frac{\rho \epsilon p \pi}{\mu(\rho+\mu)}+\frac{(1-\epsilon p) \pi}{\mu},
$$

and $E_{\mathrm{DFS}}=I_{\mathrm{DFS}}=R_{\mathrm{DFS}}=T_{\mathrm{DFS}}=0$, and an endemic state (ES) where all 90 the equilibrium components are non-zero

$$
\begin{array}{lll}
V_{\mathrm{ES}}=\frac{\epsilon p \pi}{\rho+\mu}, & S_{\mathrm{ES}}=\frac{\pi}{\mu R_{0}}, & I_{\mathrm{ES}}=\frac{\pi}{\beta}\left(R^{*}-1\right), \\
E_{\mathrm{ES}}=\frac{\sigma+\gamma+\mu}{\nu} I_{\mathrm{ES}}, & R_{\mathrm{ES}}=\frac{(1-\xi) \sigma+\gamma}{\mu} I_{\mathrm{ES}}, & T_{\mathrm{ES}}=\frac{\xi \sigma}{\mu} I_{\mathrm{ES}},
\end{array}
$$

where

$$
R^{*}=\left(1-\frac{\epsilon p \mu}{\rho+\mu}\right) R_{0}
$$

and

$$
R_{0}=\frac{\beta \nu}{(\nu+\mu)(\sigma+\gamma+\mu)} .
$$

$R^{*}$ is called the effective reproduction number, representing the expected number of secondary cases produced by a single infectious individual within a disease-naive population. It is important to note that, in a population undergoing disease prevention, $R^{*}$ depends on the level of disease susceptibility. In our case, $R^{*}$ is a function of $p$, the probability of getting vaccinated. The SEIRtype model (1) undergoes a transcritical bifurcation [45] at $R^{*}=1$. If $R^{*}>1$, then ES will be reached; otherwise, $R^{*} \leq 1$ and DFS will be reached. $R_{0}$ is the 100 basic reproduction number [45-47], obtained from the model in the absence of prevention (i.e., $p=0$ ). To quantify the impact of vaccination on epidemics, we analyze $R^{*}(p)$ given that there is an epidemic in absence of vaccination; i.e., $R_{0}>1$.

Using Eqs. (2) and (3), the endemic prevalence of the infectious disease can be written as

$$
\Pi(p)= \begin{cases}\Pi_{\mathrm{DFS}}(p), & \text { if } R^{*} \leq 1, \\ \Pi_{\mathrm{ES}}(p), & \text { if } R^{*}>1 ;\end{cases}
$$


where

$$
\Pi_{\mathrm{DFS}}(p)=\frac{I_{\mathrm{DFS}}+E_{\mathrm{DFS}}}{N_{\mathrm{DFS}}}=0,
$$

and

$$
\Pi_{\mathrm{ES}}(p)=\frac{I_{\mathrm{ES}}+E_{\mathrm{ES}}}{N_{\mathrm{ES}}}=\frac{\mu}{\beta}\left(1+\frac{\sigma+\gamma+\mu}{\nu}\right)\left(R^{*}(p)-1\right) .
$$

A critical vaccination coverage, $p_{c}$, may be defined using $R^{*}\left(p_{c}\right)=1$ or, equivalently, $\Pi_{\mathrm{ES}}\left(p_{c}\right)=0$, and verifies

$$
\epsilon p_{c}=\left(1+\frac{\rho}{\mu}\right)\left(1-\frac{1}{R_{0}}\right) .
$$

110 (i.e., $\rho=0$ and $\epsilon=1$ ), Eq. (9) recovers a well-known result; see Refs. [46, p. 87] and $[47, \mathrm{ch} .6]$.

A diagram of disease prevalence at the equilibria of the $S E I R$-type model (1), as a function of $p$, is shown in Fig. 1. ES is always stable (attracting), while 115 DFS is unstable (repelling) for $p<p_{c}$ and stable for $p>p_{c}$. It is important to note that, in the general case where the vaccination coverage is a function of time, disease-free dynamics, where $E=I=R=T=0$, is possible for all values of $R_{0}$.

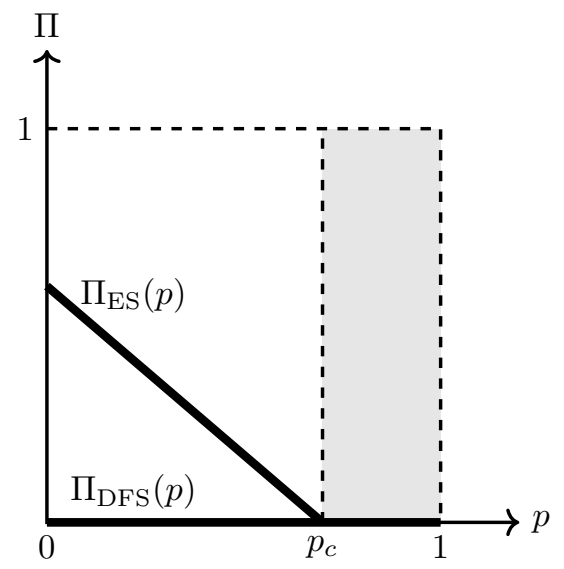

Figure 1: The endemic prevalence, $\Pi$, as a function of the vaccine coverage, $p$. If $R^{*} \leq 1$ $\left(p>p_{c}\right)$, the system reaches the disease-free state (DFS) where $\Pi_{\mathrm{DFS}}=0$. On the other hand, if $R^{*}>1\left(p<p_{c}\right)$, the system reaches the endemic state (ES) with endemic prevalence $\Pi_{\mathrm{ES}}$. We note that DFS still exists for $p<p_{c}$, but it is unstable.

\subsection{The single-player game}

120

We assume vaccination to be voluntary. The pros and cons of vaccination versus treatment perceived by a typical individual may be biased and involve monetary and/or non-monetary aspects [15] such as: price, undesired vaccine 
effects, accessibility of vaccination, vaccination schedule, time spent to get vaccinated, disease morbidity, secondary effects induced by (symptomatic or/and therapeutic) treatment, etc. In game theory, they are generally expressed as cost. A utility function is employed to make the balance of all the cost with respect to vaccination versus treatment, as described below. Game theory postulates that the individual-level decision of whether or not to get vaccinated maximizes the utility of vaccination. Mathematically, this is expressed by max-

130 imizing the utility function. As a result, we obtain the probability that a typical individual gets vaccinated, depending on cost and vaccine and epidemiological parameters. In turn, this yields the voluntary vaccination coverage. Hence, the addition of game theory to the $S E I R$-type model (1) makes explicit that the vaccination coverage is not a parameter of the model that may be easily tuned. Rather, the relative cost of vaccination versus recovery is a more tunable parameter, as we will see below.

We assume that individuals address the matter of vaccination as long as they acknowledge an epidemic threat in the absence of vaccination (i.e., $R_{0}>1$ ). Otherwise, individuals do not get vaccinated. We also assume that individuals 140 have a sense of the probability of acquiring infection when there is an epidemic threat. We express this probability using the endemic prevalence of the infectious disease, $\Pi$, defined by Eqs. (6)-(8).

The balance of cost is as follows. To prevent getting infected under epidemic threat, an individual would pay the cost of vaccination, $c_{p}$, with probability $p$ of getting vaccinated, and the cost of recovery, $c_{r}$, with probability $(1-\epsilon p) \Pi(p)$ of getting infected. A similar account of costs may be found in Ref. [23, Eqs. (2.1) and (2.2)]. The utility function of vaccination versus treatment when $R_{0}>1$ becomes:

$$
U\left(p ; c_{p}, c_{r}\right)=-p c_{p}-(1-\epsilon p) c_{r} \Pi(p),
$$

where $c_{p}$ and $c_{r}$ are positive. Introducing the relative cost of vaccination versus 150 recovery $r=c_{p} / c_{r}$ and rescaling the utility function $U(p ; r)$ by $c_{r}$, we obtain

$$
U(p ; r)=-p r-(1-\epsilon p) \Pi(p) .
$$

\section{Results}

By maximizing the utility function $U(p ; r)$ for the individual player, we obtain an expression of the probability for an individual to get vaccinated as a function of the relative cost of vaccination versus recovery. We denote this probability by $\hat{p}(r)$.

Case 1: $R^{*}>1$. The probability $\hat{p}(r)$ is a solution of $\partial U(p ; r) / \partial p=0$ and verifies

$$
\epsilon \hat{p}(r)= \begin{cases}\frac{r_{b}-r}{\tilde{r}}, & \text { if } r_{a}<r<r_{b}, \\ 0, & \text { if } r \geq r_{b},\end{cases}
$$


where

$$
\tilde{r}=\frac{2 \epsilon \mu^{2}}{(\nu+\mu)(\rho+\mu)}\left(1+\frac{\nu}{\sigma+\gamma+\mu}\right)
$$

and

$$
r_{b}=\tilde{r}\left[1-\frac{1}{2 R_{0}}+\frac{\rho}{2 \mu}\left(1-\frac{1}{R_{0}}\right)\right] .
$$

The restriction $R^{*}(\hat{p})>1$ yields $r>r_{a}$, where

$$
r_{a}=\tilde{r}\left[\frac{1}{2 R_{0}}-\frac{\rho}{2 \mu}\left(1-\frac{1}{R_{0}}\right)\right] .
$$

Equations (14) and (15) yield $r_{a}<r_{b}$ whenever $R_{0}>1$. A threshold for the probability for an individual to be effectively vaccinated is immediately obtained

$$
\epsilon \hat{p}\left(r_{a}\right)=\left(1+\frac{\rho}{\mu}\right)\left(1-\frac{1}{R_{0}}\right),
$$

retrieving Eq. (9) for $p_{c}$.

Case 2: $R^{*} \leq 1$. In this case, $0 \leq r \leq r_{a}$ and the endemic prevalence is zero; cf. Eq. (7). Utility reaches the maximum value of zero at $\hat{p}(r)=0$; cf. Eq. (11). Along with Eq. (4), this implies $R^{*}=R_{0}>1$ and leads to contradiction. We conclude that the game theoretic assumption of an equilibrium resolution of the vaccination-versus-treatment dilemma is not tenable. There exists no equilibrium coverage for voluntary vaccination once the epidemic has been averted; $\hat{p}(r)$ does not have a stable equilibrium when $R^{*} \leq 1$.

The results on voluntary vaccination coverage are summarized in Fig. 2. Given the vaccine efficacy, $\epsilon$, the domain of the function $\epsilon \hat{p}(r)$, representing the probability of effective vaccination, is divided into three regions. Region (c) corresponds to $r \geq r_{b}$, where individuals find the relative cost of vaccination versus treatment too high and do not get vaccinated; i.e., $R^{*}=R_{0}>1$. Region (b) corresponds to $r_{a}<r<r_{b}$, where some individuals adopt prevention and the epidemic is alleviated; i.e., $R_{0}>R^{*}>1$. Region (a) corresponds to $0 \leq r \leq r_{a}$ and $R^{*} \leq 1$. Individuals will get vaccinated in sufficient numbers to avert the epidemic, as long as they have the motivation to do so. However, according to our model, a long-term motivation based on disease prevalence does not exist.

This situation may be reversed if an incentive is used and individuals perceive a net gain from being vaccinated (i.e., $r$ is allowed to take negative values) when $R^{*} \leq 1$. Straightforward calculations show that, in this case, $\hat{p}(r)=1$ maximizes the utility of vaccination, independently of the perceived gain.

Figure 1, intended for the SEIR-type model (1), remains illustrative for the two-component model using game theory, as well. Just as before, ES exists for the region $0 \leq p<\hat{p}\left(r_{a}\right)=p_{c}$ (i.e., $R^{*}>1$ ) and is stable. However, DFS exists only for $p=0$ and is unstable, as we assumed $R_{0}>1$.

The equilibrium structures uncovered in Figs. 1 and 2 invite to a discussion about vaccine parameters. From the point of view of public health, the first 


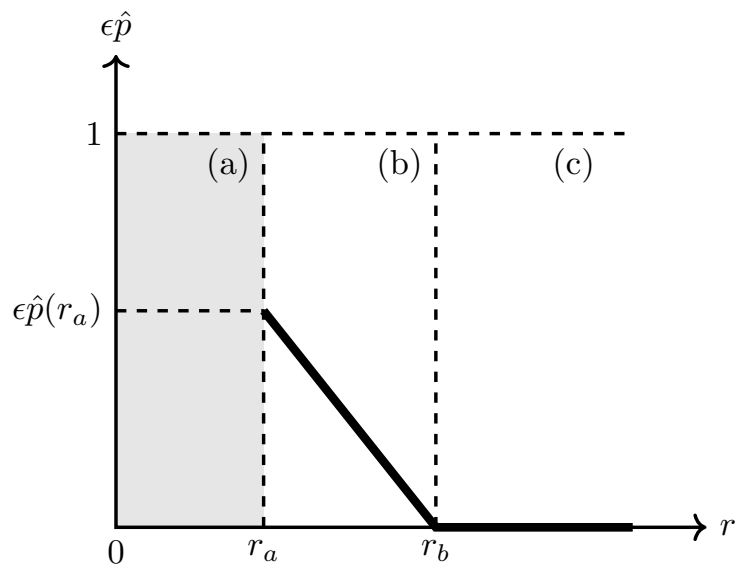

Figure 2: The probability that individuals get effectively vaccinated, $\epsilon \hat{p}(\epsilon, r)$, as a function of the relative cost of vaccination versus treatment, $r$. The domain is divided into three regions. In region (c), individuals do not get vaccinated due to the high cost of vaccination versus treatment. Region (b) corresponds to the case where lower cost encourages some individuals to adopt vaccination and, as a result, the epidemic is alleviated. For region (a), the relative cost is significantly reduced. However, the interplay between vaccination and treatment there does not lead to steady disease epidemiology.

and key desideratum is the ability to prevent epidemics; i.e., $p_{c} \leq 1$. This yields (cf. Eq. (9))

$$
\frac{\epsilon}{1+\rho / \mu} \geq 1-\frac{1}{R_{0}}
$$

and guarantees the existence of region (a) in Fig. 2, where epidemics may be temporarily prevented. From Eq. (15), we obtain that $r_{a}>0$ if and only if

$$
\frac{R_{0}-1}{\mu}<\frac{1}{\rho} .
$$

That is, region (a) exists if and only if the duration of vaccine-induced immunity, $1 / \rho$, is larger than $\left(R_{0}-1\right)$ expected lifetimes, $1 / \mu$.

The second desideratum is that region (a) be as large as possible in terms of the relative cost of vaccination versus treatment, to leave room for ample variation in cost. It can be shown that

$$
0 \leq r_{a} \leq \frac{\mu}{R_{0}(\mu+\nu)}\left(1+\frac{\nu}{\sigma+\gamma+\mu}\right) .
$$

200 Furthermore, we have $\partial r_{a} / \partial \epsilon>0$ and $\partial r_{a} / \partial \rho^{-1}>0$; i.e., $r_{a}$ increases with improving the efficacy and induced duration of immunity of the imperfect vaccine.

\subsection{Application to measles}

Measles is a very contagious infectious disease, for which only symptomatic treatment and treatment to improve disease outcomes (e.g., vitamin A) are 
available [48-50]. Recovery from infection is believed to lead to lifelong immunity [50]. However, measles can be prevented through vaccination, which induces long-term protection [51, 52]. Before the vaccine was developed, infection with measles virus was nearly universal during childhood [50]. The average number of secondary infections generated by index cases in fully susceptible populations was estimated at $R_{0}=5-18$ [46, p. 70] or 12.5-18 [42].

Mass vaccination campaigns ran in many countries once the vaccine was licensed in the 60 's, and were continued by national programs for the vaccination of children. Measles vaccine schedules varied across time and countries [51]. Initially, the recommendation was to administer one dose at 8-9 months of age.

215 Then, the recommended age for vaccination was raised to 12-15 months in some countries, mainly high-income countries, to overcome the inhibitory effect of maternal antibodies on vaccine efficacy [51]. Among children initially vaccinated after 12 months of age, vaccine efficacy is at least $95 \%$, while it can be lower for children vaccinated before 12 months of age [51]. Starting with the 1980s, most countries introduced a routine second dose of measles vaccine to further reduce the number of children left susceptible after primary vaccination and increase vaccine efficacy. Hence, the second dose is not considered as a booster [51]. The age for the second dose varies across countries: the second dose is either administrated few months apart from the first dose (e.g., France, Austria, Germany, Brazil, Australia) or a few years apart (e.g., USA, UK, Italy, Sweden, Finland), around the age of school enrollment $[51,53,54]$. The implementation of measles vaccination programs has led in many settings to the elimination of endemic transmission of measles, at least temporarily [51, 55, 56], even before adopting routine two-dose schedules [57].

We apply our modeling results to the epidemiology of measles vaccination. Because we assume that vaccination occurs shortly after birth, our results apply best for countries where the time interval between measles vaccine doses is relatively short. We propose that measles elimination for current vaccination programs is ongoing as described for region (a) in our model; see Fig. 2. ${ }_{235}$ Reaching this region was possible because, when vaccination programs were implemented, the relative cost of vaccination versus treatment (i.e., $r$ ) for measles was most certainly perceived as low by individuals, for the following main reasons: measles was endemic, parents witnessed measles-related morbidity and mortality, public health authorities would make vaccines freely available or subsidized them, and decline of measles incidence would provide direct evidence of vaccination success. However, our results also show that there is no stable equilibrium in region (a). This implies that measles epidemiology is evolving toward the border between regions (a) and (b). The transition may, however, take a long time because national vaccination programs only induce small changes in vaccination coverage, compared to mass vaccination campaigns. This transition occurs because, as high levels of coverage are achieved, individuals may perceive a larger cost of vaccination versus treatment (i.e., higher $r$ ) and lose motivation to vaccinate their children. Indeed, when measles is not endemic, most parents do not witness measles-related morbidity and mortality. Furthermore, vaccine rumors/controversies [13] may lead to vaccine hesitation [15] and 
lower vaccination coverage. A decrease below the critical coverage, $\hat{p}\left(r_{a}\right)$, may lead to epidemic resurgence $[50,58]$, and thus to a transition from region (a) to region (b), where the disease is endemic and individuals will find, once more, the motivation to vaccinate.

Our theoretical findings offer insight on vaccine parameters when the epidemic was eliminated at least temporarily through voluntary vaccination. We consider countries where measles vaccination programs have led to the elimination of measles using a one-dose routine vaccination schedule (e.g., some countries in the Americas [57]) or two doses administered in a relatively short of time (e.g., Australia [56]). In vaccination provides immunity for at least $\left(R_{0}-1\right)$ expected lifetimes. The current value of $R_{0}$ is not known, but we may assume that $R_{0}$ remains in the range of 5 to 18 , as before the vaccination campaigns. We may thus conclude that measles vaccine provides immunity for a duration much longer than the expected lifetime (4-17 times the expected lifetime). The duration of immunity of current measles vaccines is difficult to determine directly, as it requires longterm studies. A 15-year observational study in China [59] reported that the negative conversion rate of measles vaccinated individuals was $8.1-20.0 \%$ over 14 years, which leads to an estimated $63-166$ years for the duration of vaccine-

270 induced measles immunity. This is in qualitative agreement with our modeling results.

\section{Discussion}

In this paper, we used game theory and ordinary differential equations to address the dilemma of prevention versus treatment. In particular, we focused on a classic $S E I R$-type model for childhood infectious diseases subject to both vaccine prevention and treatment.

We found that voluntary vaccination may lead toward epidemic elimination if two conditions are met. First, the duration of vaccine-induced immunity should be sufficiently long; we derived a mathematical formula for this duration, ${ }_{280}$ depending on the basic reproduction number of the epidemic, $R_{0}$. Second, the relative cost of prevention versus treatment must be sufficiently low; we found a threshold cost, $r_{a}$. Disease elimination may occur when a high-performance vaccine is made available, at low cost, in an endemic setting where individuals witness disease-related morbidity and mortality, as well as the benefits of vacci285 nation, as disease incidence declines. All together, this yields a low relative cost of prevention versus treatment (i.e., lower than $r_{a}$ ), resulting in a vaccination coverage high enough to avert the epidemic. However, our modeling results show that disease epidemic elimination is only temporary; this is captured by the absence of an equilibrium for the effective coverage when $R^{*}<1$. Indeed, as vaccination coverage increases, leading to less epidemic adversity, individuals may also lose their initial motivation to vaccinate. Hence, with epidemic elimination, the perception of cost in the dilemma of prevention versus treatment may change and increase up to $r_{a}$. In turn, this causes a decrease in vaccination 
coverage and reverses disease elimination to the situation where $R^{*}=1$. Prevaccination $[16,18,60]$. However, it is very important to note that, once the epidemic is averted (in region (a)), the dynamics toward the situation where $R^{*}=1$ may be slowed down significantly, owing to continuous effort from the public health authority to maintain a low cost for vaccination.

These findings have implications for prevention and public health programs. For the condition on vaccine quality to be met, it is essential to develop one-shot highly effective vaccines that provide long-lasting immunity. For the condition on the relative cost of prevention versus treatment, we have to distinguish two epidemiological phases: the initial phase, when the vaccine is made available in presence of endemic disease, and the elimination phase, when vaccination continues after reaching high coverage. The cost for a highly effective vaccine introduced when the disease is endemic may be easily perceived as low. However, once the epidemic is eliminated, maintaining a low perceived cost for vaccination may become a complex issue, which will depend on the setting [15]. Witnessing almost no epidemic adversity, individuals may lose motivation to vaccinate. In addition, with the increased vaccination coverage, they may be particularly aware of adverse effects [61] and susceptible to vaccine rumors/controversies [13]. In this case, maintaining a low relative cost of prevention versus treatment may be a difficult and long-running task, requiring multi-scale actions.

Costs associated with vaccine accessibility and uptake, encountered by both individuals and health professionals, may act as important barriers [15] and should be reduced. They include monetary cost of the vaccines, as well as time spent on accessing vaccination, communicating about the safety profile, and administrative burden. Furthermore, vaccination incentives could be im320 plemented [62-64]. For instance, modest non-monetary incentives [63, 65] and conditional cash transfers $[62,64]$ have been used to increase the vaccination coverage. However, this type of incentives proved effective only for low-andmiddle-income settings $[62,66]$. In addition, dialogue-based interventions (e.g., social mobilization, communication through mass and social media, etc.) and 325 reminders (e.g., telephone calls or letters) have been used to encourage vaccination $[65]$.

We propose three additional interventions to maintain a low cost for vaccination. First, vaccination in high-income settings might be encouraged using health insurance policies. For instance, the health insurance provider may offer ${ }_{330}$ a progressive reduction of the insurance premium (and/or increase of benefits) along with the completion of the vaccination schedule. Second, the public health authority should acknowledge in the media the participation and success of prevention programs in search for continuous public support. Involving civil society representatives and other relevant stakeholders as full participants in vaccine 335 recommendation and policy, as it was recently done in France [67], may help improving mutual understanding and trust around vaccination. Third, recalling information about disease sequelae, and their statistics, using epidemiological data on childhood diseases from countries where vaccine coverage are low, may help individuals to perceive better the aim of prevention, and maintain a fair 
perception of prevention cost. This also requires providing clear information about vaccine adverse effects, based, for example, on statistics elaborated from the notification of adverse effects by health professionals and parents.

In addition, using the basic reproduction number, our model provides a lower bound estimate for the duration of vaccine-induced immunity against epi-

345 demic diseases controlled through vaccination. This may be a particularly important result, since the duration of vaccine-induced immunity is not precisely known for most vaccines [42]. Measuring long-standing vaccine-induced immunity (years) requires long-term follow-up of large numbers of vaccinated individuals (e.g., [59]). Nonetheless, a precise estimation of the duration of vaccine-

350 induced immunity is key to optimize immunization schedules, guide vaccination policy and enhance public trust in vaccines. Along with traditional epidemiology methods, mathematical modeling may offer valuable insight in estimating this vaccine parameter.

In conclusion, we used a game-theoretic model to discuss the dilemma of prevention versus treatment. We demonstrated the circumstances under which non-cooperative, self-interested individuals arrive to alleviate, and potentially eliminate, an epidemic through the use of an imperfect vaccine. Maintaining a low relative cost of prevention versus treatment will be the main challenge to maintain disease elimination unless incentives are considered.

360 Author contributions. R. B. conceived the model. S. J. and R. B. conducted the calculations. All authors wrote the main manuscript text, analyzed the results and have approved the final version of the article.

Declaration of interest. The authors declare no conflict of interest.

Acknowledgements. The authors thank Dominique Costagliola for helpful com365 ments on the manuscript. S. J. was supported by a Ph.D. fellowship from the French Ministry of Higher Education and Research, and the Public Health Doctoral Network coordinated by the EHESP.

\section{References}

[1] S. W. Roush, T. V. Murphy, Vaccine-Preventable Disease Table Working Group, Historical comparisons of morbidity and mortality for vaccinepreventable diseases in the United States, JAMA 298 (18) (2007) 21552163. doi:10.1001/jama.298.18.2155.

[2] F. Zhou, A. Shefer, J. Wenger, M. Messonnier, L. Y. Wang, M. Moore, T. V. Murphy, M. Cortese, L. Rodewald, A. F. Zhou, A. Shefer, Economic Evaluation of the Routine Childhood Immunization Program in the United States, 2009, Pedriatics 133 (4) (2014) 577-585. doi:10.1542/peds.20130698 .

[3] M. Johannesson, P. O. Johansson, A note on prevention versus cure, Health Policy 41 (3) (1997) 181-187. doi:10.1016/S0168-8510(97)00020-1. 
[4] P. A. Ubel, M. D. Spranca, M. L. Dekay, J. C. Hershey, D. A. Asch, Public preferences for prevention versus cure: What if an ounce of prevention is worth only an ounce of cure?, Medical Decision Making 18 (2) (1998) 141-148. doi:10.1177/0272989X9801800202.

[5] P. S. Corso, Prevention just in case or treatment just because: Measuring societal preferences, Harvard Health Policy Review 7 (2006) 32-41.

[6] R. Bosworth, T. A. Cameron, J. R. DeShazo, Is an ounce of prevention worth a pound of cure? Comparing demand for public prevention and treatment policies, Medical Decision Making 30 (4) (2010) E40-E56. doi:10.1177/0272989X10371681.

[7] D. Mortimer, L. Segal, Is the value of a life or life-year saved context specific? Further evidence from a discrete choice experiment, Cost Effectiveness and Resource Allocation : C/E 6 (2008) 8. doi:10.1186/1478-7547-6-8.

[8] D. L. B. Schwappach, The equivalence of numbers: The social value of avoiding health decline: An experimental web-based study, BMC Medical Informatics and Decision Making 2 (2002) 3. doi:10.1186/1472-6947-2-3.

[9] P. S. Corso, J. K. Hammitt, J. D. Graham, R. C. Dicker, S. J. Goldie, Assessing preferences for prevention versus treatment using willingness to pay, Medical Decision Making 22 (5 suppl) (2002) s92-s101. doi:10.1177/027298902237713.

[10] R. M. Meertens, V. M. J. de Gaar, M. Spronken, N. K. de Vries, Prevention praised, cure preferred: Results of between-subjects experimental studies comparing (monetary) appreciation for preventive and curative interventions, BMC Medical Informatics and Decision Making 13 (2013) 136. doi:10.1186/1472-6947-13-136.

[11] J. Luyten, R. Kessels, P. Goos, P. Beutels, Public preferences for prioritizing preventive and curative health care interventions: A discrete choice experiment, Value in Health 18 (2) (2015) 224-233. doi:10.1016/j.jval.2014.12.007.

[12] J. Gérvas, B. Starfield, I. Heath, Is clinical prevention better than cure?, The Lancet 372 (9654) (2008) 1997-1999. doi:10.1016/S01406736(08)61843-7.

[13] World Health Organization, Impact of rumors and crises, website accessed: March 23, 2017. URL http://vaccine-safety-training.org/impact-of-rumours-and-crises.html

[14] H. J. Larson, A. de Figueiredo, Z. Xiahong, W. S. Schulz, P. Verger, I. G. Johnston, A. R. Cook, N. S. Jones, The State of Vaccine Confidence 2016: Global Insights Through a 67-Country Survey, EBioMedicine 12 (2016) 295-301. doi:10.1016/j.ebiom.2016.08.042. 
[15] H. J. Larson, C. Jarrett, E. Eckersberger, D. M. D. Smith, P. Paterson, Understanding vaccine hesitancy around vaccines and vaccination from a global perspective: A systematic review of published literature, 2007-2012, Vaccine 32 (19) (2014) 2150-2159. doi:10.1016/j.vaccine.2014.01.081.

[16] C. T. Bauch, D. J. D. Earn, Vaccination and the theory of games, Proceedings of the National Academy of Sciences 101 (36) (2004) 13391-13394. doi:10.1073/pnas.0403823101.

[17] C. T. Bauch, A. P. Galvani, D. J. D. Earn, Group interest versus self-interest in smallpox vaccination policy, Proceedings of the National Academy of Sciences 100 (18) (2003) 10564-10567. doi:10.1073/pnas.1731324100.

[18] P. Manfredi, P. Della Posta, A. D’Onofrio, E. Salinelli, F. Centrone, C. Meo, P. Poletti, Optimal vaccination choice, vaccination games, and rational exemption: an appraisal, Vaccine 28 (1) (2009) 98-109. doi:10.1016/j.vaccine.2009.09.109.

[19] Z. Wang, M. A. Andrews, Z. X. Wu, L. Wang, C. T. Bauch, Coupled disease-behavior dynamics on complex networks: A review, Physics of Life Reviews 15 (2015) 1-29. doi:10.1016/j.plrev.2015.07.006.

[20] H. Zhang, J. Zhang, C. Zhou, M. Small, B. Wang, Hub nodes inhibit the outbreak of epidemic under voluntary vaccination, New Journal of Physics 12 (2) (2010) 23015. doi:10.1088/1367-2630/12/2/023015.

[21] H. F. Zhang, Z. X. Wu, X. K. Xu, M. Small, L. Wang, B. H. Wang, Impacts of subsidy policies on vaccination decisions in contact networks, Physical Review E 88 (1) (2013) 12813. doi:10.1103/PhysRevE.88.012813.

[22] F. Fu, D. I. Rosenbloom, L. Wang, M. A. Nowak, Imitation dynamics of vaccination behaviour on social networks, Proceedings of the Royal Society of London B: Biological Sciences 278 (1702) (2011) 42-49. doi:10.1098/rspb.2010.1107.

[23] C. T. Bauch, Imitation dynamics predict vaccinating behaviour, Proceedings of the Royal Society B: Biological Sciences 272 (1573) (2005) 16691675. doi:10.1098/rspb.2005.3153.

[24] T. C. Reluga, Game theory of social distancing in response to an epidemic, PLOS Computational Biology 6 (5) (2010) 1-9. doi:10.1371/journal.pcbi.1000793.

[25] T. C. Reluga, J. Li, Games of age-dependent prevention of chronic infections by social distancing, Journal of Mathematical Biology 66 (7) (2013) 1527-1553. doi:0.1007/s00285-012-0543-8. 
[26] T. C. Reluga, C. T. Bauch, A. P. Galvani, Evolving public perceptions and stability in vaccine uptake, Mathematical Biosciences 204 (2) (2006) 185-198. doi:10.1016/j.mbs.2006.08.015.

[27] A. P. Galvani, T. C. Reluga, G. B. Chapman, Long-standing influenza vaccination policy is in accord with individual self-interest but not with the utilitarian optimum, Proceedings of the National Academy of Sciences 104 (13) (2007) 5692-5697. doi:10.1073/pnas.0606774104.

[28] T. C. Reluga, A. P. Galvani, A general approach for population games with application to vaccination, Mathematical Biosciences 230 (2) (2011) 67-78. doi:10.1016/j.mbs.2011.01.003.

[29] J. Liu, B. F. Kochin, Y. I. Tekle, A. P. Galvani, Epidemiological game-theory dynamics of chickenpox vaccination in the USA and Israel, Journal of the Royal Society Interface 9 (66) (2012) 68-76. doi:10.1098/rsif.2011.0001.

[30] D. Sykes, J. Rychtáŕ, A game-theoretic approach to valuating toxoplasmosis vaccination strategies, Theoretical Population Biology 105 (2015) 33-38. doi:10.1016/j.tpb.2015.08.003.

[31] C. Molina, D. J. D. Earn, Game theory of pre-emptive vaccination before bioterrorism or accidental release of smallpox, Journal of the Royal Society Interface 12 (2015) 20141387. doi:10.1098/rsif.2014.1387.

[32] E. Shim, G. B. Chapman, J. P. Townsend, A. P. Galvani, The influence of altruism on influenza vaccination decisions, Journal of The Royal Society Interface 9 (74) (2012) 2234-2243. doi:10.1098/rsif.2012.0115.

[33] R. Vardavas, R. Breban, S. Blower, A universal long-term flu vaccine may not prevent severe epidemics, BMC Research Notes 3 (2010) 92. doi:10.1186/1756-0500-3-92.

[34] R. Vardavas, R. Breban, S. Blower, Can influenza epidemics be prevented by voluntary vaccination?, PLOS Computational Biology 3 (5) (2007) e85. doi:10.1371/journal.pcbi.0030085.

[35] R. Breban, Health newscasts for increasing influenza vaccination coverage: An inductive reasoning game approach, PLOS One 6 (12) (2011) e2830010. doi:10.1371/journal.pone.0028300.

[36] R. Breban, R. Vardavas, S. Blower, Mean-field analysis of an inductive reasoning game: Application to influenza vaccination, Physical Review E 76 (3) (2007) 31127. doi:10.1103/PhysRevE.76.031127.

[37] S. Bhattacharyya, C. T. Bauch, R. Breban, Role of word-of-mouth for programs of voluntary vaccination: A game-theoretic approach, Mathematical Biosciences 269 (2015) 130-134. doi:10.1016/j.mbs.2015.08.023. 
[38] S. Bhattacharyya, C. T. Bauch, "Wait and see" vaccinating behaviour during a pandemic: a game theoretic analysis, Vaccine 29 (33) (2011) 55195525 .

[39] B. Wu, F. Fu, L. Wang, Imperfect vaccine aggravates the long-standing dilemma of voluntary vaccination, PLoS One 6 (6) (2011) e20577. doi:10.1371/journal.pone.0020577.

[40] F. Verelst, L. Willem, P. Beutels, Behavioural change models for infectious disease transmission: A systematic review (2010-2015), Journal of the Royal Society Interface 13 (125). doi:10.1098/rsif.2016.0820.

[41] A. R. Mclean, S. M. Blower, Imperfect Vaccines and Herd Immunity to HIV, Proceedings of the Royal Society B: Biological Sciences 253 (1336) (1993) 9-13. doi:10.1098/rspb.1993.0075.

[42] S. A. Plotkin, W. A. Orenstein, P. A. Offit, Vaccines: Sixth Edition, Elsevier, University of Pennsylvania, Philadelphia, United States, 2012.

[43] M. Ng, N. Fullman, J. L. Dieleman, A. D. Flaxman, C. J. L. Murray, S. S. Lim, Effective Coverage: A Metric for Monitoring Universal Health Coverage, PLoS Medicine 11 (9). doi:10.1371/journal.pmed.1001730.

[44] K. E. Colson, P. Zúñiga-Brenes, D. Ríos-Zertuche, C. J. Conde-Glez, M. C. Gagnier, E. Palmisano, D. Ranganathan, G. Usmanova, B. Salvatierra, A. Nazar, I. Tristao, E. Sanchez Monin, B. W. Anderson, A. Haakenstad, T. Murphy, S. Lim, B. Hernandez, R. Lozano, E. Iriarte, A. H. Mokdad, Comparative estimates of crude and effective coverage of measles immunization in low-resource settings: Findings from Salud Mesoamérica 2015, PLoS ONE 10 (7) (2015) 1-21. doi:10.1371/journal.pone.0130697.

[45] P. van den Driessche, J. Watmough, Further notes on the basic reproduction number, in: F. Brauer, P. van den Driessche, J. Wu (Eds.), Mathematical Epidemiology, Lecture notes in Mathematics, Springer Berlin Heidelberg, Berlin, Heidelberg, 2008, Ch. 6, pp. 159-178. doi:10.1007/978-3-540-789116 .

[46] R. M. Anderson, R. M. May, Infectious Diseases of Humans: Dynamics and Control, Oxford University Press, 1991.

[47] O. Diekmann, H. J. A. P. Heesterbeek, Mathematical Epidemiology of Infectious Diseases: Model Building, Analysis and Interpretation, Wiley Series in Mathematical and Computational Biology, Wiley, 2000.

[48] World Health Organization, Measles vaccines: WHO position paper, Weekly epidemiological record 84 (35) (2009) 349-360. doi:10.1016/j.biologicals.2009.04.005. 
[49] Centers for Disease Control Prevention, Measles (Rubeola), website accessed: July 28, 2017 (2017).

URL https://www.cdc.gov/measles/hcp/index.html

[50] Centers for Disease Control Prevention, Epidemiology and prevention of vaccine-preventable diseases: Measles, website accessed: April 27, 2017 (2017).

URL https://www.cdc.gov/vaccines/pubs/pinkbook/meas.html

[51] P. M. Strebel, M. J. Papania, A. P. Fiebelkorn, N. A. Halsey, Measles vaccine, in: Vaccines (Sixth Edition), Elsevier, London, 2013, pp. $352-$ 387. doi:10.1016/B978-1-4557-0090-5.00028-8.

[52] J. C. Watson, S. C. Hadler, C. a. Dykewicz, S. Reef, L. Phillips, Measles, mumps, and rubella-vaccine use and strategies for elimination of measles, rubella, and congenital rubella syndrome and control of mumps: recommendations of the Advisory Committee on Immunization Practices (ACIP), MMWR. Recommendations and reports : Morbidity and mortality weekly report. Recommendations and reports / Centers for Disease Control 47 (RR-8) (1998) 1-57.

[53] Bundesministerium für Gesundheit und Frauen, Impfplan Österreich, Wien, [English translation: Austrian vaccination plan, published by the Federal Ministry of Health and Women, Vienna, 2017] (2017).

[54] European Centre for Disease Prevention and Control, Vaccine Schedule, website accessed: August 9, 2017.

URL http://vaccine-schedule.ecdc.europa.eu/Pages/Scheduler.aspx

[55] H. Peltola, S. Jokinen, M. Paunio, T. Hovi, I. Davidkin, Measles, mumps, and rubella in Finland: 25 years of a nationwide elimination programme, The Lancet Infectious Diseases 8 (12) (2008) 796-803. doi:10.1016/S14733099(08)70282-2.

[56] H. F. Gidding, N. V. Martin, V. Stambos, T. Tran, A. Dey, G. K. Dowse, H. A. Kelly, D. N. Durrheim, S. B. Lambert, Verification of measles elimination in Australia: Application of World Health Organization regional guidelines, Journal of Epidemiology and Global Health 6 (3) (2015) 197209. doi:10.1016/j.jegh.2015.12.004.

[57] A. E. Sever, J. J. Rainey, E. R. Zell, K. Hennessey, A. Uzicanin, C. CastilloSolórzano, V. Dietz, Measles elimination in the Americas: A comparison between countries with a one-dose and two-dose routine vaccination schedule, Journal of Infectious Diseases 204 (SUPPL. 2). doi:10.1093/infdis/jir445.

[58] D. R. Q. Lemos, A. R. Franco, M. L. F. de Sá Roriz, A. K. B. Carneiro, M. H. de Oliveira Garcia, F. L. de Souza, R. Duron Andino, L. P. de Góes Cavalcanti, Measles epidemic in Brazil in the post-elimination period: Coordinated response and containment strategies, Vaccine 35 (13) (2017) 1721-1728. doi:10.1016/j.vaccine.2017.02.023. 
[59] D. Bin, Z. H. Chen, Q. C. Liu, T. Wu, C. Y. Guo, X. Z. Wang, H. H. Fang, Y. Z. Xiang, Duration of immunity following immunization with live measles vaccine: 15 years of observation in Zhejiang Province, China, Bulletin of the World Health Organization 69 (4) (1991) 415-423.

[60] P. Doutor, P. Rodrigues, M. do Céu Soares, F. A. C. C. Chalub, Optimal vaccination strategies and rational behaviour in seasonal epidemics, Journal of Mathematical Biology 73 (6) (2016) 1437-1465. doi:10.1007/s00285-0160997-1.

[61] L. Serpell, J. Green, Parental decision-making in childhood vaccination, Vaccine 24 (19) (2006) 4041-4046. doi:10.1016/j.vaccine.2006.02.037.

[62] E. Owusu-Addo, R. Cross, The impact of conditional cash transfers on child health in low- and middle-income countries: A systematic review, International Journal of Public Health 59 (4) (2014) 609-618. doi:10.1007/s00038014-0570-x.

[63] A. V. Banerjee, E. Duflo, R. Glennerster, D. Kothari, Improving immunisation coverage in rural India: clustered randomised controlled evaluation of immunisation campaigns with and without incentives, BMJ (Clinical research ed.) 340 (9655) (2010) c2220. doi:10.1136/bmj.c2220.

[64] Australian Government Department of Health, No Jab, No Pay - New Immunisation Requirements for Family Assistance Payments (2015).

[65] C. Jarrett, R. Wilson, M. O'Leary, E. Eckersberger, H. J. Larson, the SAGE Working Group on Vaccine Hesitancy, Strategies for addressing vaccine hesitancy - A systematic review, Vaccine 33 (34) (2015) 4180-4190. doi:10.1016/j.vaccine.2015.04.040.

[66] J. Adams, R. J. McNaughton, S. Wigham, D. Flynn, L. Ternent, J. Shucksmith, Acceptability of parental financial incentives and quasimandatory interventions for preschool vaccinations: Triangulation of findings from three linked studies, PLoS ONE 11 (6) (2016) 1-14. doi:10.1371/journal.pone.0156843.

[67] Rapport de la Concertation Citoyenne sur la Vaccination, website accessed: April 10, 2017 (2016).

URL http://concertation-vaccination.fr/la-restitution/ 